\title{
The relationship between inhibitory control and speech production in young multilinguals
}

\author{
Iga Krzysik \\ Adam Mickiewicz University, Poznań \\ ikrzysik@wa.amu.edu.pl
}

\begin{abstract}
Speech production in multilinguals involves constant inhibition of the languages currently not in use. In relation to phonological development, higher inhibitory skills may lead to the improved suppression of interference from the remaining languages in one's repertoire and more accurate production of target features. The participants were 20 sequential multilingual learners (13-year-olds with L1 Polish, L2 English, L3 German), acquiring their L2 and L3 by formal instruction in a primary school. Inhibition was measured in a modified flanker task (Eriksen \& Eriksen 1974; Poarch \& Bialystok 2015). Multilingual production of voice onset time (VOT) and rhotic consonants was tested in a delayed repetition task (e.g. Kopečková et al. 2016; Krzysik 2019) in their L2 and L3. The results revealed that higher inhibitory control was related to increased global accuracy in the L2 and L3 production. Moreover, higher inhibitory control was also linked to higher accuracy in the overall L2 production, but there was no significant relationship with the L3 accuracy. These findings suggest that inhibition may play a role in phonological speech production, however, it may depend on one's level of proficiency.
\end{abstract}

Keywords: Speech production; inhibitory control; phonological development; multilingualism.

\section{Introduction}

Multilingual processing and production requires constant monitoring and suppression of remaining languages in the participant's repertoire in order to accurately perceive and produce linguistic input. An ability to inhibit irrelevant information and responses has been related to improved linguistic performance in a host of studies across various domains (e.g. Gollan et al. 2011; Linck et al. 2012; Mercier et al. 2014; Poarch \& Bialystok 2015; Lev-Ari \& 
Peperkamp 2013; Darcy et al. 2016). Despite the previous contributions, the relationship between inhibitory control and multilingual phonological processing can still be described as an understudied domain. The aim of current study is to investigate inhibitory control in the context of phonological accuracy in multilingual speech production.

\subsection{Inhibitory control and phonological language processing}

Inhibitory control has been defined as an ability to suppress irrelevant information and prepotent responses. Miyake et al. (2000) identified inhibition as one of the components of executive functions (along with shifting and updating). In a subsequent study, Miyake et al. (2012) changed its status to a correlate of the common executive function - the ability to maintain and use task goals and goal related information. Furthermore, Friedman \& Miyake (2004) offered a subdivision of the components of inhibitory control, differentiating between prepotent response inhibition (suppressing automatic responses), resistance to distractor interference (suppressing distracting information) and resistance to PI (preventing memory intrusions from formerly relevant information). Such classification of the described subcomponents requires also a diversification of the task and measures. In the context of bi/multilingual language processing, inhibition is described as a mechanism of language control which lessens or prevents the activation of a given non-target language (its lexicon, phonological features etc.). However, the underlying characteristics of this mechanism and its possible subtypes, governing inhibitory control in various aspects of language processing, are still not well-understood (as demonstrated by Borragan et al. 2018). One of the existing conceptualisations, a seminal inhibitory control model proposed by Green (1986) and subsequently updated by Green \& Wei (2014) assumes that the languages of a bi/ multilingual have varying levels of activation which affect the level of influence on the language currently in use. The languages operate using a activation-inhibition mechanism and can be divided into three groups; the selected language currently in use with highest level of activation, the activated language(s) which affect the current processing and dormant languages which do not participate in the processing. The levels of activation can be modified by such factors as the frequency and the recency of use of a given language. Moreover, the model suggest that the more activated a language is, the more inhibitory control is required to suppress it (De Angelis 2007: 73-74). Consequently, 
the accuracy in language perception and production as well as the extent of cross-linguistic influence may be influenced by one's inhibitory capacity. Despite the fact that several weaknesses of the model have been pointed out (e.g. Wrembel 2015: 60-61), it still constitutes a starting point for further discussions of the role of inhibition in language processing. Inhibitory control has been investigated in numerous studies exploring its relation to various aspects of linguistic performance and experience such as language switching (e.g. Costa and Sansebastan 2004; Costa et al. 2006; Calabria et al. 2012; Declerck \& Philipp 2015; Liu et al. 2019), language activation (e.g. Linck et al. 2008; Giezen et al. 2015), differences between monolingual and bi/multilingual processing (e.g. Bialystok et al. 2005; Blumenfeld \& Marian 2011; Poarch \& Bialystok 2015).Despite the wealth of previous research spanning across various domains, the relation between inhibitory control and the L2 (Ln) phonology still constitutes an understudied research area. Several studies employing measures of inhibitory control along with the speech perception and production tasks have already indicated that greater inhibitory control may be linked to the improved discrimination of features and the production of target-like realisations. For instance, Lev-Ari \& Peperkamp (2013) explored the potential role of inhibitory control in the process of the $\mathrm{L} 2$ perception and production in late English-French sequential bilinguals residing in France. The participants completed a retrieval induced inhibition task and a sentence reading supplemented by a free conversation and a phoneme categorisation tasks in separate English and French sessions. The results indicated that the lower inhibitory control was linked to a more French-like perception and production of Voice Onset Time of voiceless English stops. Consequently, the participants with stronger inhibitory control were more likely to succeed in suppressing the regressive influence of their L2 on their L1. Another study investigating the modalities of perception and production in relation to inhibition was conducted by Mora \& Darcy (2013). Three groups of language learners (L1 Spanish learners of English, L1 Catalan-Spanish learners of English and L1 English learners of Spanish) completed a delayed sentence repetition task, ABX categorization task and a measure of inhibition, a retrieval-induced inhibition task. The outcomes of the study indicated the inhibitory control contributed to an increased accuracy in perception for the sequential bilinguals but not for the simultaneous bilinguals. These results suggest that the inhibitory mechanisms may differ as a function of proficiency. Darcy et al. (2014) examined the role of inhibitory control in the L2 phonological perception and production in L1Spanish/L2-English learners and L1-English/L2-Spanish learners. The results 
revealed that stronger inhibitory control in a speeded set-switching task was related to improved perception accuracy in an ABX task for both groups, however, no clear pattern concerning production accuracy in a delayed sentence repetition task was observed. A subsequent study by Darcy et al. (2016) further investigated the possible relationship of inhibitory control and speech perception / production on a larger sample of the L1-Spanish/L2-English learners and L1-English/L2-Spanish learners. The analysis revealed that stronger inhibitory control was related to greater accuracy in the L2 segmental perception as well as the L 2 consonant production accuracy. A perception / production study by Sigmeth et al. (2019) further investigated the role of inhibitory control in the multilingual processing of L1 German learners of L2 English and L3 Polish. The production results obtained in the L2 ABX task showed a significant correlation with the inhibitory capacity of the participants. However, no such relationship was observed for the L2 production of the selected vowels and consonants obtained by means of a delayed repetition task. A study by Sigmeth $\&$ Golin (2018), focusing predominantly on perception, examined a group of adolescent L1 German learners of L2 English and L3 Polish was tested longitudinally on their inhibitory control by means of a modified flanker task and their speech perception in a forced-choice task. No relationship between inhibitory control and perception was found across the two tested languages, however, the participants' inhibition scores improved over time. These developmental findings may suggest that the multilingual exposure and a continuous experience of switching and suppressing the languages not in use may enhance the inhibitory capacity of learners. Despite the fact that the studies reviewed above did not produce uniform results, they can allow for the formulation of the tentative implications for the further research on the relationship of phonological production/perception and inhibition: (1) stronger inhibitory control may contribute to the successful suppression of the language currently not in use, (2) stronger inhibitory control may minimise cross-linguistic influence, (3) stronger inhibitory control may improve the processing of the relevant acoustic cues in the L2/L3 input and lead to more accurate production of features.

\subsection{The present study}

Previous studies have positioned inhibitory control as an important factor in bilingual language processing, with some possible implications for phonological speech perception and production. However, the mechanism of language 
inhibition, especially in relation to phonological development, is still not fully understood and definitely requires further empirical examination. The present study will explore the relationship between inhibitory control and speech production, but it will also expand the scope to multilingual speakers, namely, adolescent sequential multilingual learners (L1 - Polish, L2 - English, L3 German). The research questions posed in the current study are as follows: (1) what is the relation between the inhibitory control and speech production in adolescent multilingual learners, (2) what is the role of language status (understood as the L2 vs. L3) and how does it affect the role of inhibition in phonological production? On the basis of the results obtained in the previous research, it can be hypothesised that stronger inhibitory control will be related to the increased accuracy in speech production in adolescent sequential multilingual learners. The scarcity of resources does not allow for drawing extensive hypotheses about the relationship between the L2, L3 and inhibitory control being modified by the language status (L2 vs. L3). However, the role of learning experience and language dominance hierarchy as factors potentially affecting the strength of the relationship between the tested languages and inhibitory control measures constitutes one of the subjects of inquiry. The findings were obtained by means of two tasks: a delayed repetition task measuring speech production in the L2 and L3 and a widely applied flanker task, assessing the ability to suppress irrelevant stimuli and accounting for inhibitory capacity. In order to control for the possible effects of language history, exposure and proficiency, the participants completed a language history and use questionnaire.

\subsection{Focal phonological features in the present study}

The focal phonological features of interest in the present study (outlined in Table 1) were determined by contrasting the systems of Polish, English and German (Jassem 2003; Malisz \& Żygis 2015; Cruttenden 2014; Beckman et al. 2013; Lein et al. 2016; Dittmers et al. 2017). The two focal features in this study, namely voice onset time (VOT) in voiceless stops and rhotics, were selected based on the comparison of the systems of L1 Polish, L2 English and L3 German. VOT parameter describes the time interval between the release of a plosive and the onset of the vocal cord vibration (Rojczyk 2011: 37). L1 Polish is characterised by a prevoiced VOT pattern for voiced stops and a short lag VOT for voiceless stops. L2 English and German show similar patterns - 
a short lag VOT for voiced stops and a long lag VOT for voiceless stops. Rhotics across these languages display even more variance; L1 Polish features an alveolar trill or flap, L2 English a postalveolar approximant and L3 German a uvular fricative or trill. The selection of such contrastive features across the studied languages was intended to highlight the participants' phonological sensitivity exhibited in the accuracy of speech production in theirL2 and L3. The previous studies investigating the development of these features in the production of the L1-Polish learners (e.g. Waniek-Klimczak 2005; Krzysik 2019) indicated that both the acquisition of non-native VOT patterns and rhotic consonants is highly variable and may exhibit complex patterns. Several studies have also demonstrated that VOT patterns may indicate the levels of language activation and inform about the strength of inhibition of the nontarget lag in voiceless plosives (Fricke et al. 2015; Jacobs et al. 2016) e.g. by the presence or absence of the L1 patterns in L2 speech. Such characteristics render the selected features an interesting research material for the present study.

Table 1. Focal features across the the L1, L2 and L3 of the participants.

\begin{tabular}{lll}
\hline Language & Rhotic consonant & VOT of voiceless plosives \\
\hline L1 - Polish & alveolar trill or flap & short lag \\
L2 - English & postalveolar approximant & long lag \\
L3 - German & uvular fricative or trill & long lag \\
\hline
\end{tabular}

\section{Method}

\subsection{Participants}

The participants involved 20 adolescent multilingual learners (mean age = 12.65, $\mathrm{SD}=0.48$; female to male ratio $=10: 10)$, L1 native speakers of Polish, acquiring English as their L2 (with 7 years of formal instruction) and German as their L3 (after 1.5 months of formal instruction) in the formal context of a primary state school in the western part of Poland. All the participants were enrolled in the same class and received the same amount of foreign language instruction from the same teachers, who were non-native speakers of the taught languages. The amount of foreign language instruction in the L2 amounted to 
$3 \times 45$ minutes per week, whereas in the L3 to $2 \times 45$ minutes. The initial sample of 27 participants was reduced to 20 students on the basis of the following exclusion criteria: a different L1, simultaneous bilingualism, a different order of foreign language acquisition and a more advanced self-declared experience and proficiency in English and German.

\subsection{Testing session}

The testing session took place on the premises of the school in a quiet room; each student was tested individually. Speech production and inhibition tasks described below were administered as a part of a larger battery of tests examining phonological development of multilinguals in a longitudinal project. The tasks of the testing battery were presented in L2 and L3 blocks, counterbalanced across the participants. Each block started with a short discussion in the respective language in order to induce language mode. The data analysed in the present paper was obtained at the first testing session (T1) of the said project, conducted in late October and early November of 2018. The informed consent was obtained both from the parents/legal guardians and the participants. The study was positively evaluated by the ethics committee at Adam Mickiewicz University, Poznań.

\subsection{Inhibitory control task}

Inhibitory control was assessed by means of a flanker task (Eriksen \& Eriksen 1974, based on the modified version proposed by Poarch \& Bialystok 2015). Consequently, it has to be asserted that the study focuses primarily on one component of inhibitory control, namely, the resistance to distractor interference/interference suppression (Friedman \& Miyake 2004; Luk et al. 2010). The selection of the tasks reflects the research questions of the present study, focusing on the mechanism affecting the production of the accurate phonological categories and its relationship with mechanisms potentially supressing undesirable interference from other languages. This non-linguistic measure was also selected to decrease the linguistic load already present in the battery of tasks in the larger study, justified by no clear advantage to the language based tasks. Finally, the measure was selected for its previous successful applications in the studies involving children or adolescents (Poarch \& Bialystok 2015; 
Sigmeth \& Golin 2018). The modified flanker task employed in the present study involved a decision about the direction of the middle arrow displayed on the computer screen in 4 types of speeded trials: neutral, baseline trials, congruent and incongruent. Baseline trials displayed a single arrow in the middle of the screen, whereas neutral trials displayed an arrow surrounded by two diamond shaped figures. In congruent trials, the middle arrow was surrounded by the arrows pointing in the same direction. In incongruent trials, the middle arrow was surrounded by the arrows pointing in a different direction. In relation to the model task the proportions were shifted to focus on the congruent and incongruent trials. The task did not feature the no-go block as it was predominantly concerned with the interference suppression, i.e. distractor interference represented by the incongruent trials (Luk et al. 2010) and their relation to the congruent trials. The task in the present study featured four blocks: a neutral block, a baseline block, and two congruent and incongruent blocks. The trials within each block were randomised. Each trial started with a $500 \mathrm{~ms}$ fixation before the stimulus onset. The time allotted for the response was 3000 $\mathrm{ms}$ after the stimulus onset. The trials were terminated by a motor response. The responses were collected with a button box. In the present study, the score of inhibition derived from the modified flanker task was be based on the reaction time difference between the incongruent and congruent trials. The obtained "flanker effect score" indicated the strength of inhibitory control.

\subsection{Speech production task in the L2 and L3}

Speech production in the L2 and L3 was measured by means of a delayed repetition tasks. The task featured a sequence of pre-recorded mini-dialogues including token words with the focal phonological features. Each mini-dialogue consisted of two sentences. Sentence 1. included a word with a given target feature. Sentence 2. served as an intervening material, which was aimed at reducing the possibility of direct imitation and enabling the access to the speaker's representations of the tested features. The template for the L2 version of the task is provided below:

Sentence 1. I say *target word with the embedded feature* again.

Sentence 2. What do you say?

Participants' response: I say *target word with the embedded feature* again. 
The mini-dialogues were pre-recorded by the native speakers of the L2 (Standard Southern British English) and the L3 (Standard German). The words with the embedded features used in the tasks were common nouns (the full list is available in Appendix 1). Both the task in the L2 and the L3 featured six items with VOT in voiceless plosives and 4 items with rhotic consonants. The selection of the target words was predominantly based on the learning materials of the participants. The L2 and L3 version of the task were administered separately, after the participants were introduced into the respective language modes by means of a short conversation. The participants were requested to listen to the mini-dialogues through a pair of semi-open headphones and repeat the sentences with the embedded target words right after each pre-recorded sequence ended. The participants' renditions were recorded using a dynamic microphone plugged to a computer via an external soundcard (two channel recordings, 16bit, $44.1 \mathrm{kHz}$ ). Both the $\mathrm{L} 2$ and $\mathrm{L} 3$ task included also other contrastive features selected as a part of a larger, longitudinal project.

\section{Results}

\subsection{Inhibitory control task}

Following the criteria outlined by Poarch \& Bialystok (2015), mean reaction time scores and mean accuracy rates were calculated for each condition of flanker task. The participants whose RTs or accuracy scores were $2.5 \mathrm{SD}$ below or above the mean were excluded from the analysis. Two participants were identified as outliers. Flanker scores indicating the strength of the participants' inhibitory control were derived from the RT data of the correctly completed congruent and incongruent trials; the scores were calculated by subtracting the RTs obtained in the congruent trials from those obtained in the incongruent trials. Low scores indicated that the time required for providing an accurate response on the congruent and incongruent trials was comparable. The suppression of the incongruent stimuli required less cognitive resources; such results were linked to a greater degree of inhibitory control. Higher scores suggested that a participant required more time to complete an incongruent trial; suppression of the incongruent material required a greater effort. 
Table 2. Flanker task RT means by the type of trial and flanker score.

\begin{tabular}{llll}
\hline Language & $\mathrm{N}$ & Rhotic consonant & SD \\
\hline Congruent trial RTs & 18 & 492.6 & 55.5 \\
Incongruent trial RTs & 18 & 552.2 & 68.5 \\
Flanker score & 18 & 59.5 & 19.1 \\
\hline
\end{tabular}

\subsection{Speech production task}

The recordings of the VOT tokens obtained in the delayed repetition tasks in the L2 and L3 were evaluated in the process of acoustic analysis. VOT of the initial voiceless stops in the L2 and L3 realisations was measured from the onset of the stop release to the onset of the voicing of the subsequent vowel using Praat software (Boersma \& Weenink 2019). (VOT means obtained for each token are available in Appendix 2.) The results of the participants were compared to the target realisations from the stimuli pre-recorded by the native speakers of the L2 and L3. The intervals established for a correct answer were 20 milliseconds above and below the VOT of the target realisation. A realisation falling $20 \mathrm{~ms}$ below the target was regarded as a hybrid realisation (an intermediated stage between L1-like VOT and target-like VOT) and granted 0.5 points. Target-like realisations were identified within the bounds of $10 \mathrm{~ms}$ below and $20 \mathrm{~ms}$ above the target and awarded 1 point. Non-target-like realisations and mispronunciations (understood as a production of a different feature) were awarded 0 accuracy points. The total (global) number of accuracy points was 12; the participants could have scored a maximum of 6 VOT accuracy points per each language version of the task. Consequently, the amount of points possible to score mirrored the number of the VOT tokens in each language version of the task.

Figure 1. shows the distribution of accuracy scores by language and feature according to the categories established above. The majority of the participants' realisations were nontarget like (the total of $67 \%$ ), which indicates a substantial reliance on the L1 categories during speech production in the L2 and L3. Hybrid realisations, understood as intermediate categories between the non-target and target-like VOT constituted $15 \%$ of the total scores for both languages; such results may indicate an ongoing process of establishing VOT values of foreign language contexts. Target-like realisations amounted to 9\% of the scores, which may suggest that the acquisition of aspirated voiceless 
plosives may pose a great difficulty, even in the more proficient L2. The final category of mispronunciations constituted $10 \%$ of all realisations; the majority of erroneous realisations concerned the L3 (7\%), which reflects the short experience and a limited exposure at the onset of the third language instruction.

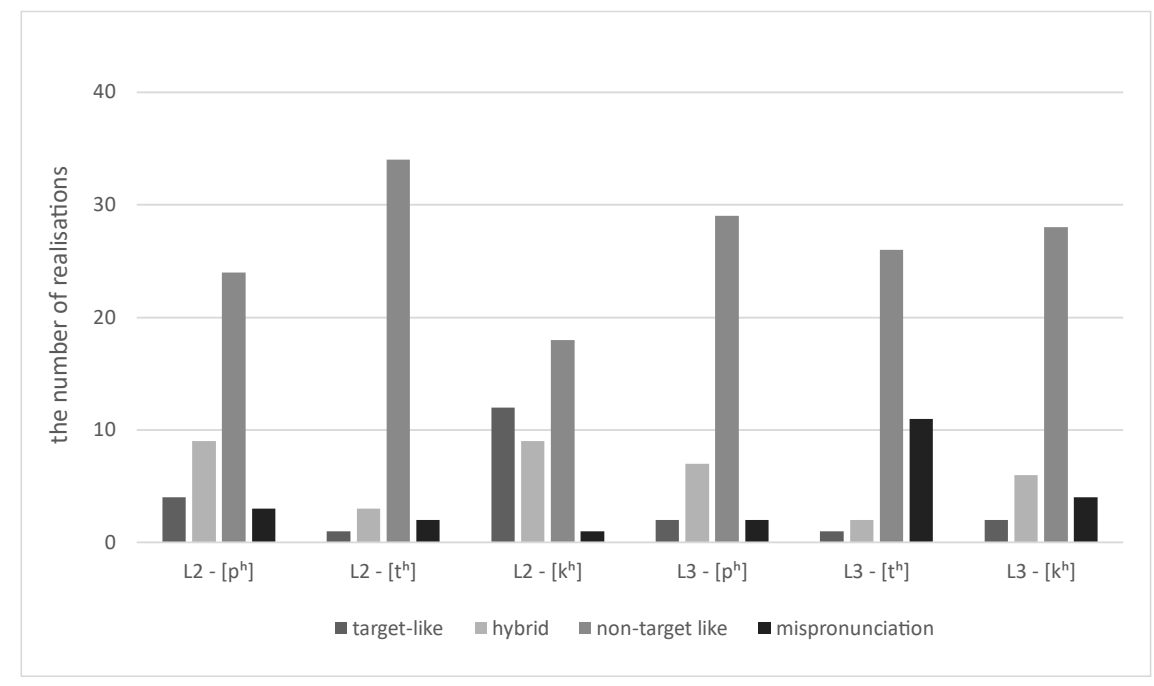

Figure 1. The distribution of accuracy scores in delayed repetition task in L1 and L2.

Table 3 presents the mean accuracy scores obtained across the features globally and separately for the L2 and L3 (taking into account two participants excluded from the analysis based on their results on the modified flanker tasks). The overall mean accuracy scores for the L2 and L3 showed that the acquisition of VOT patterns was at an initial stage. Despite the 7 years of formal instruction in the L2, the native-like representations of the aspirated voiceless plosives values were not fully established; the participants exhibited a mixture of target-like, hybrid and L1-based VOT values. The L3 productions were mostly L1-based, with infrequent instances of target-like production. When it comes to the production of the individual features, the participants were the most accurate in the production of $\left[\mathrm{k}^{\mathrm{h}}\right]$, followed by $\left[\mathrm{p}^{\mathrm{h}}\right]$ in their $\mathrm{L} 2$. A similar pattern was observed by Waniek-Klimczak (2005) in L1-Polish, L2English late bilinguals, who produced [ $\left.\mathrm{k}^{\mathrm{h}}\right]$ with longer, more native-like VOT. 
In the $\mathrm{L} 3$, the accuracy levels for $\left[\mathrm{k}^{\mathrm{h}}\right]$ and $\left[\mathrm{p}^{\mathrm{h}}\right]$ were the closely comparable. The category of $\left[\mathrm{t}^{\mathrm{t}}\right]$ was identified as the least accurate in both languages.

Table 3 . VOT accuracy scores globally and by language for all tokens.

\begin{tabular}{lcccccc}
\hline VOT accuracy & N & Mean & SD & Min & Max & Number of tokens \\
\hline L2 accuracy & 18 & 1.44 & 1.26 & 0 & 4 & 6 \\
L3 accuracy & 18 & 0.66 & 0.98 & 0 & 4 & 6 \\
\hline
\end{tabular}

The recordings of the rhotic consonants tokens obtained in the delayed repetition tasks in L2 and L3 were evaluated by two independent, phonetically trained raters, who were instructed on the features of interest and the methods of scoring (outlined also in the previous paper by Krzysik 2019). The raters were requested to rate the participants' production of the rhotic consonants in their L2 and L3 either as accurate (awarded 1 point) or inaccurate (awarded 0 points). The accuracy was operationalised as a target-like production of the rhotic consonants in the L2 (as postalveolar approximants) and the L3 (as uvular fricatives or trills) whereas inaccuracy encompassed non-target. Inter-rater reliability was calculated using Cohen's kappa $(\mathrm{k}=0.8, \mathrm{p}<.05)$, which indicated a substantial agreement between the raters. The realisations assessed incongruently by two raters were evaluated by an additional rater; in these cases the prevailing rating was favoured. The total number of evaluated items was 8 , i.e. 4 per each language version of the task. Consequently, the maximum global score amassed to 8 points. Accuracy scores for the production of rhotic consonants are presented in Table 4.

Table 4. Rhotics accuracy scores globally and by language for all tokens.

\begin{tabular}{lcccccc}
\hline Rhotics accuracy & N & Mean & SD & Min & Max & Number of tokens \\
\hline L2 accuracy & 18 & 1.38 & 1.29 & 0 & 4 & 4 \\
L3 accuracy & 18 & 0.67 & 1.34 & 0 & 4 & 4 \\
\hline
\end{tabular}

In order to prepare the accuracy scores for the subsequent analysis in relation to inhibitory control, the following scores for VOT and rhotics were computed: 
a global accuracy score in L2 and L3 production and overall L2 and L3 scores. The scores are presented in Table 5.

Table 5. Speech production accuracy scores globally and by language for all tokens.

\begin{tabular}{lcccccc}
\hline $\begin{array}{l}\text { Overall production } \\
\text { accuracy }\end{array}$ & $\mathrm{N}$ & Mean & SD & Min & Max & Number of tokens \\
\hline $\begin{array}{l}\text { Global L2 } \\
\text { and L3 accuracy }\end{array}$ & 18 & 4.16 & 1.93 & 2 & 8.5 & 20 \\
L2 accuracy & 18 & 2.83 & 1.55 & 1 & 7.5 & 10 \\
L3 accuracy & 18 & 1.33 & 1.67 & 0 & 5.5 & 10 \\
\hline
\end{tabular}

\subsection{Inhibitory control and speech production}

It was hypothesised that stronger inhibitory control will be related to the increased accuracy in speech production of adolescent sequential trilinguals. Consequently, it was assumed that the participants who scored higher on inhibitory control would also have higher scores on the L2 and L3 production measure, as their representations of phonological features were supposedly more in line with the target models. This relationship was examined by means of computing Pearson's correlation coefficient between the three accuracy datasets obtained in the speech production tasks (accuracy in the L2, accuracy in the L3 and the global accuracy scores) and the flanker score computed on the basis of incongruent and congruent trial RTs. It is worth noting that the improvement in speech production is signified by the increasing results in the accuracy scores, whereas in inhibitory control it is marked by a decrease in the flanker scores. Therefore, the correlation reflecting a meaningful relationship between inhibitory control and speech production is expected to be negative. Pearson correlation coefficient computed for the flanker scores and the global accuracy scores for the L2 and L3 revealed a substantial negative correlation $(\mathrm{r}=-.69, \mathrm{~N}=18, \mathrm{p}=.002$ ) (Figure 2). The correlation between the flanker scores and the accuracy scores in the L2 can be described as moderate $(\mathrm{r}=$ $-.47, \mathrm{~N}=18, \mathrm{p}=0.05$ ) (Figure 3 ). The relationship between the obtained 
flanker scores and the accuracy in the L3 was also negative, however, statistically insignificant $(\mathrm{r}=-.37, \mathrm{~N}=18, \mathrm{p}=0.135)$. The obtained results suggest that inhibitory control may influence the accuracy of speech production. These findings suggest that inhibition may play a substantial role in the process of phonological speech production, however, its influence may be modified by the language status (L2 vs. L3), proficiency and length of acquisition.

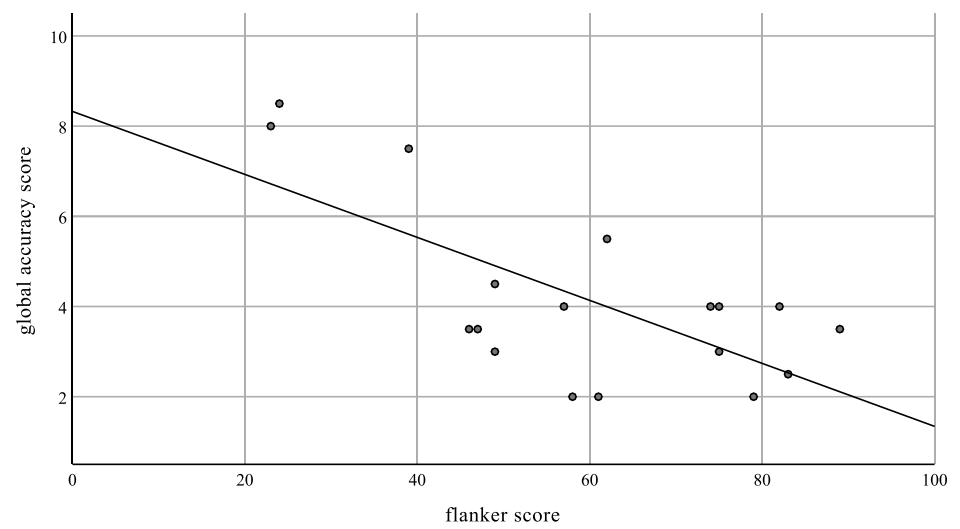

Figure 2. The relationship between the flanker scores and the global accuracy scores.

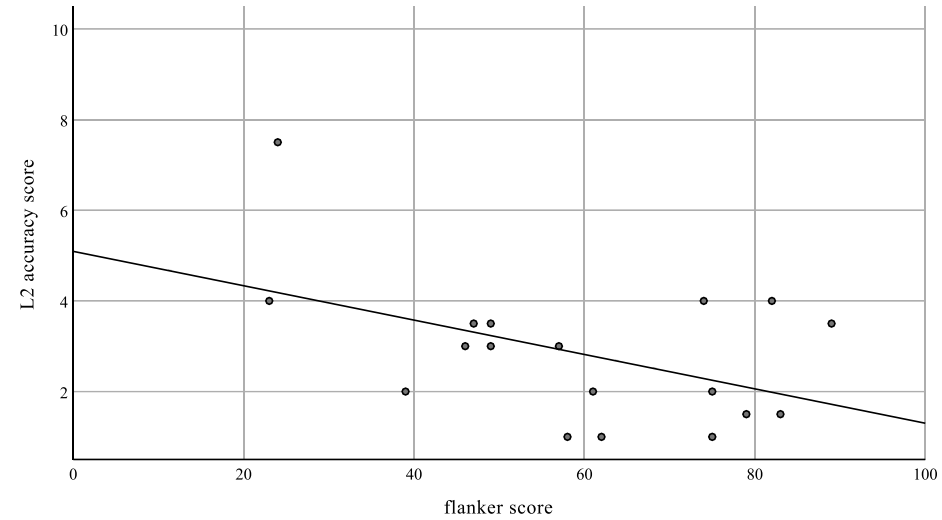

Figure 3. The relationship between the flanker scores and L2 accuracy scores. 


\section{Discussion}

Previous studies into the relationship between inhibitory control and speech perception/production indicated that stronger inhibitory control may facilitate the processes associated with the successful suppression of the language currently not in use. Moreover, stronger inhibitory control may be linked to the minimised negative cross-linguistic influence and the improved processing of the relevant acoustic cues in the foreign language input, leading to a more accurate perception and production of the $\mathrm{Ln}$ features.

The present study investigated the hypothesised relationship between the inhibitory control and speech production of young, sequential multilinguals. The inhibitory capacity of the participants was tested in a modified flanker task, whereas their speech production was elicited in a delayed repetition task in the L2 and L3. The obtained results revealed a substantially significant relationship between inhibitory control and the global accuracy in speech production in the L2 and L3. The correlations of inhibitory control and separate language scores might have possibly been modified by the language status (L2 vs. L3). The L2 of the participants (defined by a longer and possibly more robust language experience and more established representations) exhibited a moderately significant relationship with the inhibitory control scores. The L3 (with a shorter learning experience, less established representations) did not show such a relationship. These results may indicate that inhibitory control has possibly influenced the phonological representations in the $\mathrm{L} 2$ and is reflected in its relationship with the L2 production accuracy. The phonological representations in the L3 have just started forming, therefore the role of mechanisms of inhibitory control tested in the study might be less prominent (as indicated by the lack of significant correlation).

The obtained findings correspond with the results of the previous studies, which have suggested that stronger inhibitory control may facilitate the suppression of the languages currently not in use in the context of speech production (Lev-Ari \& Peperkamp 2013; Mora \& Darcy 2013; Darcy et al. 2016). Moreover, as the previously conducted research suggested, stronger inhibitory control was associated with a greater production accuracy. The results of the present study can also be related to the previous findings suggesting that proficiency may moderate the role of inhibitory control in language processing or determine the inhibitory mechanisms used. The study by Mora \& Darcy (2013) revealed that individual differences may be neutralised in balanced bilinguals due to the constant practice of switching and suppressing. Consequently, the 
role of inhibition may not be fully detectable in the low proficiency individuals (L3 in the case of the participants in the present study) due to a possibly different set of executive function mechanisms at play during their speech production. The findings obtained for the L2 may be discussed in relation to the inhibitory component of resistance to distractor interference/interference suppression; the improved control over the interfering material in the modified flanker task was associated with the better accuracy of speech production, possibly related to the successful inhibition of the features of other languages. The results of the study may be also related to the predictions of Green's inhibitory model; the dominant status of the L1 of the participants had an immense influence on their production and manifested in the percentages of inaccurate, L1derived phonological realisations. Based on the said model, it can be hypothesised that the frequently used and the most proficient L1 remained highly activated as a background to the selected L2 or L3.

The results of the L2 and L3 speech production tasks employed in the study may also have some implications for the formal language-learning environment. The accuracy scores for VOT and rhotic consonants, especially in the context of the L2, suggest that the amount or the characteristics of language input (e.g. in-class focus on some other aspects of language than pronunciation) delivered via formal instruction may not be sufficient for establishing more native-like pronunciation patterns.

Further examination of the relationship between inhibitory control and speech production requires the analysis of the remaining production data collected in the broader context of the longitudinal study (such as the remaining phonological features in the delayed repetition tasks). In order to maintain sensitivity to the developmental nature of multilingual acquisition of phonology in the study, the ratings and accuracy measures may further maintain and introduce intermediate, hybrid categories. Furthermore, the analysis of the collected speech perception data may provide some valuable additional insights into the nature of the investigated relationship, as inhibition was linked with speech production in a number of previous studies. Consequently, it may possibly introduce yet another field of comparison with the previous studies (e.g. Sigmeth \& Golin 2018; Sigmeth et al. 2019). Additionally, the comparative analysis of the longitudinal data is vital to account for the role of changing proficiency as a factor affecting the role of inhibitory control in speech production and perception. Moreover, a more detailed approach to the analysis may also be beneficial; replacing global accuracy categories with feature- 
based categories may provide a more accurate outline of multilingual proficiency. Further research directions must be also informed by the shifting approach to the methodology, which departs from the comparisons of bilingual linguistic performance with only one selected cognitive task (visible in the studies by e.g. Green \& Abutalebi 2013; Bialystok et al. 2015; Baum \& Titone 2014; Borragan et al. 2016; Bialystok 2017; Beatty Martínez \& Dussias 2019). Future studies would offer a broader outlook by introducing additional measures of different subcomponents of inhibitory control.

The present study intended to explore the relationship between the inhibitory control and speech production by focusing on the underresearched multilingual population. The results indicated a substantially significant relationship between the inhibitory control measured in a modified flanker task and the phonological accuracy in a speech production task, however, the relation was affected by language proficiency and status. Further research is required to establish the mechanisms underlying the role of inhibition in phonological language processing and its links with other factors influencing this process.

\section{Ethics statement}

Informed consent was obtained from the parents/legal guardians and the participants. The study was positively evaluated by the ethics committee at Adam Mickiewicz University, Poznań.

\section{Acknowledgements}

This research is a part of a larger project entitled: Individual differences in multilingual acquisition of phonology: Longitudinal study on young learners (grant reference number: 2017/27/N/HS2/00285), funded by National Science Centre, Poland. I would like to thank the anonymous reviewers for their comments and insights which improved the quality of the paper. I would also like to thank my supervisor dr hab. Magdalena Wrembel, prof UAM, for her guidance and valuable suggestions. 


\section{References}

Beckman, J., M. Jessen \& C. Ringen. 2013. Empirical evidence for laryngeal features: Aspirating vs. true voice languages. Journal of Linguistics 49. 259-284.

Baum, S. \& D. Titone. 2014. Moving toward a neuroplasticity view of bilingualism, executive control, and aging. Applied Psycholinguistics 35(5). 857-894.

Beatty-Martínez, A.L. \& P.E. Dussias. 2019. Revisiting masculine and feminine grammatical gender in Spanish: linguistic, psycholinguistic, and neurolinguistic evidence. Frontiers in Psychology 10.

Bialystok, E., M. Martin \& M. Viswanathan. 2005. Bilingualism across the lifespan: The rise and fall of inhibitory control. International Journal of Bilingualism 9. $103-119$.

Bialystok, E., J.F. Kroll, D.W. Green, B. MacWhinney \& F.I.M. Craik. 2015. Publication bias and the validity of evidence: What's the connection? Psychological Science 26(6). 944- 946.

Bialystok E. 2017. The bilingual adaptation: How minds accommodate experience. Psychological Bulletin 143(3). 233-262.

Blumenfeld, H.K. \& V. Marian. 2011. Bilingualism influences inhibitory control in auditory comprehension. Cognition 118(2). 245-257.

Boersma, P. \& D. Weenink. 2019. Praat: doing phonetics by computer [Computer program]. Version 6.1.08, retrieved 5 December 2019 from http://www.praat.org/.

Borragan, M., C.D. Martin, A. de Bruin \& J.A. Duñabeitia. 2018. Exploring different types of inhibition during bilingual language production. Frontiers in Psychology 9.

Calabria, M., M. Hernandez, F.M. Branzi \& A. Costa. 2012. Qualitative differences between bilingual language control and executive control: Evidence from taskswitching. Frontiers in Psychology 2.

Costa, A. \& M. Santesteban. 2004. Lexical access in bilingual speech production: evidence from language switching in highly proficient bilinguals and L2 learners. Journal of Memory and Language 50. 491-511.

Costa, A., M. Santesteban \& I. Ivanova. 2006. How do highly proficient bilinguals control their lexicalization process? Inhibitory and language-specific selection mechanisms are both functional. Journal of Experimental Psychology: Learning Memory and Cognition 32. 1057-1074.

Cruttenden, A. 2014. Gimson's pronunciation of English. Oxford: Routledge.

Darcy, I. \& J.C. Mora. 2013. Inhibition and phonological processing in a second language. A presentation delivered at the conference of European Second Language Association, Amsterdam 2013.

Darcy, I., J.C. Mora \& D. Daidone. 2016. Attention control and inhibition influence phonological development in a second language. Proceedings of the International Symposium on the Acquisition of Second Language Speech (Concordia Working Papers in Applied Linguistics 5). 115-129.

Darcy, I., J.C. Mora \& D. Daidone. 2016. The role of inhibitory control in second language phonological processing. Language Learning 66. 741-773.

De Angelis, G. 2007. Third or additional language acquisition. Clevendon: Multilingual Matters. 
Declerck, M. \& A.M. Philipp. 2015. A review of control processes and their locus in language switching. Psychonomic Bulletin Review 22. 1630-1645.

Dittmers, T., C. Gabriel, M. Krausel \& S. Topal. 2017. Positive transfer from the heritage language? The case of VOT in German/Turkish and German/Russian learners of L3 French, Russian, and English. A presentation delivered at the Workshop on Multilingual Language Acquisition, Processing and Use, Poznań 2017.

Eriksen, B.A. \& C.W. Eriksen. 1974. Effects of noise letters upon identification of a target letter in a non-search task. Perception and Psychophysics 16. 143-149.

Fricke, M., J.F. Kroll \& P.E. Dussias. 2016. Phonetic variation in bilingual speech: A lens for studying the production-comprehension link. Journal of Memory and Language 89. 110-113.

Friedman, N.P. \& A. Miyake. 2004. The relations among inhibition and interference control functions: a latent-variable analysis. Journal of Experimental Psychology: General 133(1). 101-135.

Giezen, M.R., H.K. Blumenfeld, A. Shook, V. Marian \& K. Emmorey. 2015. Parallel language activation and inhibitory control in bimodal bilinguals. Cognition 141. 9-25.

Green, D. 1986. Control, activation, and resource: A framework and a model for the control of speech in bilinguals. Brain and Language 27. 210-223.

Green, D.W. \& J. Abutalebi. 2013. Language control in bilinguals: The adaptive control hypothesis. Journal of Cognitive Psychology 25(5). 515-530.

Green, D. \& W. Li. 2014. A control process model of code-switching. Language, Cognition and Neuroscience 29(4). 499-511.

Gollan, T.H., T. Sandoval \& D.P. Salmon. 2011. Cross-language intrusion errors in aging bilinguals reveal the link between executive control and language selection. Psychological Science 22(9). 1155-1164.

Jacobs, A., M. Fricke \& J.F. Kroll. 2016. Cross-language activation begins during speech planning and extends into second language speech. Language Learning 66(2). 324-353.

Jassem, W. 2003. Illustrations of the IPA: Polish. Journal of the International Phonetic Association 33(1). 103-107.

Kopečková, R., M. Marecka, M. Wrembel \& U. Gut. 2016. Interactions between three phonological subsystems of young multilinguals: the influence of language status. International Journal of Multilingualism 13(4). 426-443.

Lev-Ari, S. \& S. Peperkamp. 2013. Low inhibitory skill leads to non-native perception and production in bilinguals' native language. Journal of Phonetics 41(5). 320331.

Lein, T., T. Kupisch \& J. van de Weijer. 2016. Voice onset time and global foreign accent in German-French simultaneous bilinguals during adulthood. International Journal of Bilingualism 20(6). 732-749.

Li, P., S. Sepanski \& X. Zhao. 2006. Language history questionnaires: A web-based interface for bilingual research. Behavioral Research Methods 38. 202-210.

Liu, C., C.-L. Yang, L. Jiao, J.W. Schwieter, X. Sun \& R. Wang. 2019. Training in language switching facilitates bilinguals' monitoring and inhibitory control. Frontiers in Psychology 10. 
Linck, J.A., N. Hoshino \& J.F. Kroll. 2008. Cross-language lexical processes and inhibitory control. The Mental Lexicon 3(3). 349-374.

Linck, J.A., J.W. Schwieter \& G. Sundermand. 2012. Inhibitory control predicts language switching performance in trilingual speech production. Bilingualism: Language and Cognition 15(3). 651-662.

Luk, G., J.A. Anderson, F.I. Craik, C. Grady \& E. Bialystok. 2010. Distinct neural correlates for two types of inhibition in bilinguals: Response inhibition versus interference suppression. Brain and Cognition 74(3). 347-357.

Malisz, Z. \& M. Żygis. 2015. Voicing in Polish: Interactions with lexical stress and focus. Proceedings of the 18th of International Congresses of Phonetic Sciences. London: International Phonetic Association.

Mercier, J., I. Pivneva \& D. Titone. 2014. Individual differences in inhibitory control relate to bilingual spoken word processing. Bilingualism: Language and Cognition 17(1). 89-117.

Miyake, A., N.P. Friedman, M.J. Emerson, A.H. Witzki, A. Howerter \& T.D. Wager. 2000. The unity and diversity of executive functions and their contributions to complex frontal lobe tasks: A latent variable analysis. Cognitive Psychology 41(1). 49-100.

Miyake, A. \& N.P. Friedman. 2012. The nature and organization of individual differences in executive functions: Four general conclusions. Current Directions in Psychological Science 21(1). 8-14.

Poarch, G.J. \& E. Bialystok. 2015. Bilingualism as a model for multitasking. Developmental Review 35. 113-124.

Rojczyk, A. 2011. Perception of the English Voice Onset Time continuum by Polish learners. In J. Arabski \& A. Wojtaszek (eds.), The acquisition of L2 phonology, 37-58. Bristol: Multilingual Matters.

Sigmeth, K. \& C. Golin. 2018. „Executive function and phonological perception in young L3 learners. Paper presented at Young Linguists’ Meeting 2018, Poznań.

Sigmeth, K., G. Poarch \& R. Kopečková. 2019. Phonological acquisition and inhibitory control in L2 English learners. A presentation delivered at Conference on Multilingualism 2019, Leiden.

Waniek-Klimczak, E. 2011. Aspiration in Polish: A sound change in progress? In M. Pawlak \& J. Bielak (eds.), New perspectives in language, discourse and translation studies. Berlin: Springer-Verlag. 3-12.

Wrembel, M. 2015. In search of a new perspective: Cross-linguistic influence in the acquisition of third language phonology. Poznań: Wydawnictwo Naukowe UAM.

\section{Corresponding author:}

Iga Krzysik

Faculty of English

Adam Mickiewicz University

Grunwaldzka 6

60-780 Poznań

Poland

ikrzysik@wa.amu.edu.pl 


\section{Appendix 1}

VOT tokens in the L2 and L3 delayed repetition tasks.

\begin{tabular}{lll}
\hline & L2 & L3 \\
\hline 1. & pot & Pott \\
2. & party & packe \\
3. & toss & toll \\
4. & target & Tante \\
5. & cot & Koch \\
6. & carpet & Kappe \\
\hline
\end{tabular}

Rhotics tokens in the L2 and L3 delayed repetition tasks.

\begin{tabular}{lll}
\hline & L2 & L3 \\
\hline 1. & Rucksack & rabbit \\
2. & Reise & hurry \\
3. & rette & mirror \\
4. & Lehrer & rose \\
\hline
\end{tabular}

\section{Appendix 2}

VOT measurement means for the voiceless plosives in the L2 and L3 with the error rates (for 20 realizations) accounting for the mispronunciations (understood as the instances of substitutions with a different feature).

\begin{tabular}{llccc}
\hline Plosive & Token word & VOT $(\mathrm{ms})$ & SD & Error rate $\%$ \\
\hline$\left[\mathrm{p}^{\mathrm{h}}\right]$ & pot & 35 & 15.2 & $15 \%$ \\
{$\left[\mathrm{p}^{\mathrm{h}}\right]$} & party & 33 & 19.8 & $0 \%$ \\
{$\left[\mathrm{t}^{\mathrm{h}}\right]$} & toss & 40 & 11.7 & $0 \%$ \\
{$\left[\mathrm{t}^{\mathrm{h}}\right]$} & target & 42 & 15.9 & $5 \%$ \\
{$\left[\mathrm{k}^{\mathrm{h}}\right]$} & cot & 60 & 13.8 & $0 \%$ \\
{$\left[\mathrm{k}^{\mathrm{h}}\right]$} & carpet & 62 & 20.5 & $5 \%$ \\
{$\left[\mathrm{p}^{\mathrm{h}}\right]$} & Pott & 31 & 11.8 & $5 \%$ \\
{$\left[\mathrm{p}^{\mathrm{h}}\right]$} & packe & 30 & 14.2 & $5 \%$ \\
{$\left[\mathrm{t}^{\mathrm{h}}\right]$} & toll & 37 & 17.1 & $35 \%$ \\
{$\left[\mathrm{t}^{\mathrm{h}}\right]$} & Tante & 32 & 16.3 & $10 \%$ \\
{$\left[\mathrm{k}^{\mathrm{h}}\right]$} & Koch & 55 & 12.8 & $10 \%$ \\
{$\left[\mathrm{k}^{\mathrm{h}}\right]$} & Kappe & 50 & 14.0 & $15 \%$ \\
\hline
\end{tabular}

